\title{
Similar Degree in Mechanical Left Ventricular Dyssynchrony Between Right Ventricular Outflow Tract and Right Ventricular Apical Pacing: A Strain Doppler Imaging Study
}

\author{
Young Soo Lee, $\mathrm{MD}^{1}$, Seong Wook Han, $\mathrm{MD}^{2}$, Yoon Nyun Kim, $\mathrm{MD}^{2}$, Chang Wook Nam, $\mathrm{MD}^{2}$, \\ Hyung Sub Kim, MD ${ }^{2}$, Kee Sik Kim, MD ${ }^{1}$ and Robert W. Rho, $\mathrm{MD}^{3}$ \\ ${ }^{1}$ Department of Cardiology, Catholic University of Daegu, ${ }^{2}$ Keimyung University Dongsan Medical Center, Daegu, Korea \\ ${ }^{3}$ University of Washington School of Medicine, Seattle, Washington, USA
}

\section{ABSTRACT}

Background and Objectives: Long-term right ventricular (RV) apex pacing has been associated with left ventricular (LV) systolic dysfunction. However, pacing in the RV outflow tract (RVOT) is associated with a narrower QRS duration and may have a more normal LV activation in comparison to RV apical (RVA) pacing. We hypothesized that RVOT pacing is associated with less mechanical dyssynchrony compared to RVA pacing and that it also more closely resembles mechanical activation in normal controls with a narrow QRS. Subjects and Methods: We studied 9 patients with RV pacing, 9 with left bundle branch block (LBBB), and 15 normal controls with a narrow QRS. In the RV pacing group, we paced from the RVA and RVOT. At the end of each pacing train, we obtained echocardiographic images in the apical 4- and 2-chamber views and obtained the following parameters: the compression/expansion crossover point (CEP) for myocardial strain and the time from QRS onset to the CEP in the strain image. The degree of dyssynchrony was evaluated using the dispersion and standard deviation of CEP times in 12 segments of the LV. Results: Significant dyssynchrony was observed in the RVOT pacing group compared to the group with normal QRS. No significant difference was observed in LV mechanical dyssynchrony among the RVOT pacing, RVA pacing, and LBBB groups. Conclusion: RVOT pacing is associated with significant LV dyssynchrony. Although the RVOT has been recommended as an alternative site for pacing, this approach may have adverse effects on long-term LV function. (Korean Circ J 2008;38:590-595)

KEY WORDS: Cardiac pacing, artificial; Right ventricle.

\section{Introduction}

The objective of cardiac pacing is to optimize cardiac performance. This is dependent on three main parameters: the chronotropic function of the sinus node or pacemaker, the quality of atrioventricular synchrony across the spectrum of rates and global inotropic states as governed by autonomic tone, and the intraventricular activation sequence. The advent of intravenous lead delivery systems established the right ventricular apex (RVA) as the standard site for pacemaker lead implanta-

\section{Received: June 18, 2008}

Revision Received: August 11, 2008

Accepted: August 29, 2008

Correspondence: Seong-Wook Han, MD, Department of Internal Medicine,

School of Medicine, Keimyung University Dongsan Medical Center, 194

Dongsan-dong, Jung-gu, Daegu 700-712, Korea

Tel: 82-53-250-7411, Fax: 82-53-250-7034

E-mail:swhan@dsmc.or.kr tion. However, several studies have demonstrated the deleterious consequences of RVA pacing in patients with cardiomyopathy. ${ }^{12)}$ Abnormal conduction of the paced depolarization through the ventricular myocardium results in dyssynchronous left ventricular (LV) contraction due to slow intramuscular conduction, in comparison to brisk conduction through the His-Purkinje system. The net result is impaired systolic and diastolic function. Furthermore, LV dyssynchrony may cause myocardial perfusion abnormalities that further diminish cardiovascular efficiency and function. ${ }^{4) 5}$

These observations have led to an interest in selecting new RV pacing sites in order to achieve a more physiological pattern of ventricular activation. ${ }^{6) 7)}$ Therefore, other sites have been proposed for RV pacing lead implantation: the RV outflow tract (RVOT), ${ }^{8) 9}$ RV free wall, ${ }^{10)}$ and His bundle area. ${ }^{11)}$ The ventricular activation sequence observed in RVOT pacing may more closely resemble that occurring during normal His-Purkinje ac- 
tivation. We hypothesized that RVOT pacing is associated with less mechanical dyssynchrony compared to RVA pacing and that it more closely resembles mechanical activation in normal control patients with a narrow QRS.

\section{Subjects and Methods}

\section{Patient population}

We enrolled 9 patients who underwent radiofrequency catheter ablation for supraventricular tachycardia (SVT) and who had normal LV systolic function and no regional wall motion abnormalities noted during echocardiography performed before the procedure; these subjects served as the study group. We also enrolled 9 patients with idiopathic left bundle branch block (LBBB) with a QRS duration of $>120$ msec. Fifteen normal subjects were included as controls. All patients gave informed consent before enrollment in the study.

\section{Study design}

Nine patients were brought to the EP lab for electrophysiologic study and ablation of SVT. After completing the radiofrequency catheter ablation for SVT, pacing was performed via a temporary pacing catheter (Biosense Webster, Diamond Bar, CA, USA, 2-5 mm interval) positioned under fluoroscopic guidance in the RVA and then in the RVOT. Pacing was performed at each site at threshold with a cycle length of $500 \mathrm{msec}$ for a duration of 3 minutes. Echocardiographic myocardial images were stored with color coding at the end of each pacing train. The time interval between pacing trains was 10 minutes. Echocardiographic myocardial images were also obtained in two sets of controls, including patients with idiopathic LBBB and patients with normal QRS duration. We evaluated and compared the degree of LV contraction and dyssynchrony in four groups of patients: 1. RVA pacing, 2. RVOT pacing, 3. patients with LBBB in normal sinus rhythm (NSR), and 4. patients with normal QRS in NSR. We excluded the patients in whom we could not measure the Doppler parameters in stored images. In all patients, the QRS width was measured as the widest among all the leads.

\section{Echocardiography}

Each individual underwent a full echocardiographic examination before and after the procedure using a Vingmed Vivid 7 (General Electric, Horten, Norway) system. The images were stored digitally on a commercially available software system (Echopac 6.3.6; GE Vingmed, Horten, Norway) and analyzed offline. The echocardiograms were analyzed in pairs with the reporter blinded to the data of each scan. The LV ejection

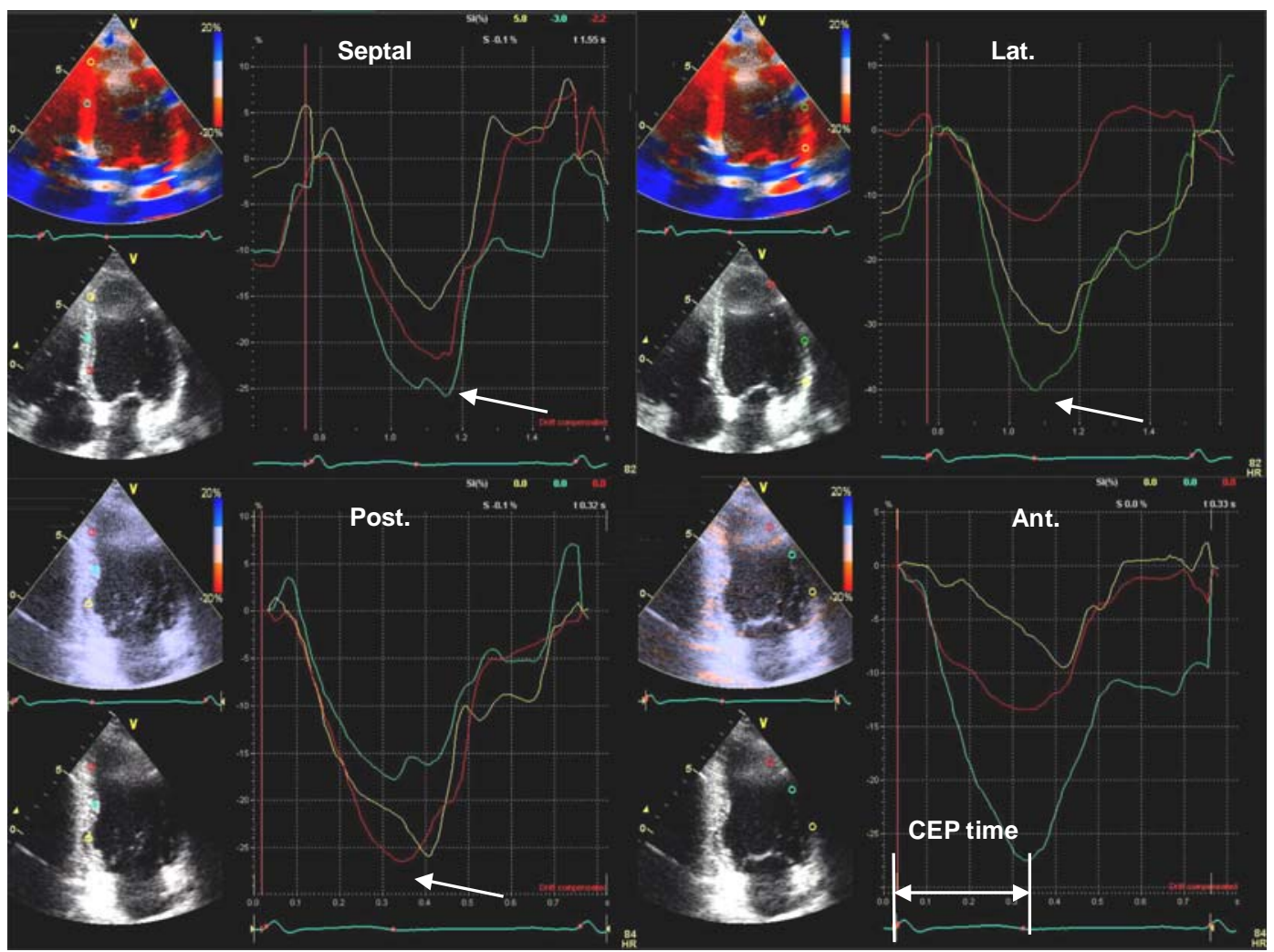

Fig. 1. Measurement of strain in twelve segments. The strain was measured at the compression/expansion crossover point (arrow), which represented the degree of actual contraction. We also defined the time from QRS onset to the compression/expansion crossover point (CEP) as the CEP time (arrow). Since this CEP time represents the actual contraction time of each segment, it could be a useful tool for analyzing dyssynchrony. 
fraction was assessed using Simpson's biplane equation for calculating volumes. The LV diameter and mass were measured in the parasternal long axis view using $\mathrm{M}$ mode. Tissue Doppler color imaging was performed using the standard apical 4- and 2-chamber views for the long-axis motion of the LV with a frame rate of over 100/sec. At least 3 consecutive beats in each view were stored and digitized for offline analysis. The mean data of three beats was used for the final analysis. From the apical 4- and 2-chamber views, four different LV wall segments-namely the septal, lateral, inferior, and anterior segments-were selected, and then three sample volumes of $5 \mathrm{~mm}$ were positioned at the basal, middle, and apical segments in each wall (Fig. 1). The myocardial strain profile was reconstituted from the tissue Doppler color images. The echocardiographic examination was performed with the same method in the resting state in the normal and LBBB groups.

The myocardial systolic strain was measured at the compression/expansion crossover point (CEP) (Fig. 1), which represented the degree of actual contraction of the LV. We also measured the time from the QRS onset to the CEP, which was defined as the CEP time. Since this CEP time represents the exact time-point of the actual contraction time of each segment, it is a useful tool for analyzing dyssynchrony. ${ }^{12) 13)}$ The degree of LV dyssynchrony was represented by the dispersion and standard deviation (SD) of the CEP times. The dispersion was obtained by calculating the difference between the maximum and minimum CEP times from all studied segments. In addition, the dispersion was corrected for the heart rate (HR) using the Bazett formula ${ }^{14)}$ \{corrected dispersion $\left(\mathrm{msec}^{1 / 2}\right)=$ dispersion $\left.(\mathrm{msec}) / \mathrm{HR}(\mathrm{msec})^{1 / 2}\right\}$, because the dispersion could be affected by the heart rate. The inter- and intra-observer concordances of CEP time were $88 \%$ and $92 \%$, respectively.

\section{Statistical analysis}

The SPSS 12.0 (SPSS, Inc., Chicago, Illinois) statistical software package was used for all calculations. Data are presented as means \pm SDs for continuous variables and as percentages for the categorical data. Differences between groups were analyzed using the analysis of variance
(ANOVA) test. Categorical data were analyzed using the Chi-square test. $\mathrm{P}<0.05$ were regarded as statistically significant.

\section{Results}

\section{Demographic characteristics}

Table 1 presents the demographic characteristics of the study population. The LBBB group was older and had larger LV mass and chamber size, with lower ejection fraction (Table 1).

\section{QRS width}

The QRS duration of normal and pre-pacing beats was significantly shorter than that for the RVOT and RVA pacing and LBBB groups. There was no significant difference in QRS duration among the RVA pacing, RVOT pacing, and LBBB groups (Fig. 2).

\section{Degree of left ventricular contraction}

The mean strain was similar for the pacing and LBBB groups and was significantly lower than that seen in the normal control group (Fig. 3). In terms of sub-analysis of the strain in each wall among the groups, the strain in the septal wall in the normal group was significantly higher. However, in the pacing and LBBB groups, we could not find the prominent area of strain (Fig. 4).

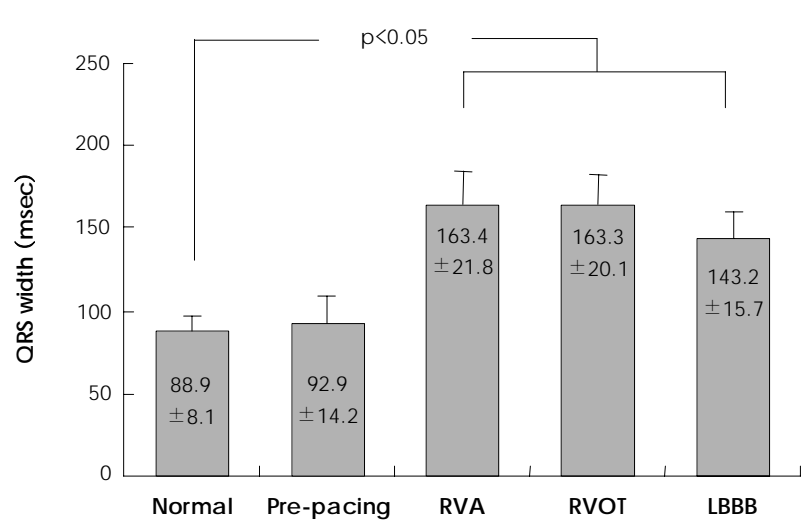

Fig. 2. Comparison of the QRS width in each group. RVA/RVOT: right ventricular apical and outflow tract pacing, LBBB: left bundle branch block.

Table 1. Characteristics of the study population

\begin{tabular}{|c|c|c|c|c|}
\hline & Normal & RV pacing & LBBB & $\mathrm{p}$ \\
\hline No. of patients & 15 & 9 & 9 & \\
\hline Male (\%) & $13(86.7 \%)$ & $4(44.4 \%)$ & $2(22.2 \%)$ & 0.005 \\
\hline Age (years) & $36.1 \pm 10.8$ & $45.7 \pm 17.1$ & $68.4 \pm 7.3^{* \dagger}$ & $<0.0001$ \\
\hline $\operatorname{LVDd}(\mathrm{cm})$ & $5.12 \pm 0.40$ & $4.79 \pm 0.54$ & $5.38 \pm 0.48^{* \dagger}$ & 0.002 \\
\hline LVDs (cm) & $3.22 \pm 0.31$ & $3.12 \pm 0.30$ & $3.78 \pm 0.57^{* \dagger}$ & 0.037 \\
\hline $\operatorname{LVEF}(\%)$ & $65.0 \pm 4.20$ & $63.0 \pm 8.01$ & $54.8 \pm 9.83^{*}$ & 0.014 \\
\hline LV mass (g) & $161.7 \pm 28.76$ & $128.3 \pm 26.18^{*}$ & $203.4 \pm 38.32^{*} \dagger$ & $<0.001$ \\
\hline
\end{tabular}

*<0.05 compared to the normal group, $\dagger<0.05$ compared to the RV pacing group. RV: right ventricle, LBBB: left bundle branch block, LVEF: left ventricular ejection fraction, LVDd/s: diastolic/systolic left ventricle dimension 


\section{Degree of left ventricular dyssynchrony}

In terms of the changes in the total contraction time, the mean CEP time corrected for heart rate was similar between the RV pacing and LBBB groups and was significantly higher than that in the normal control

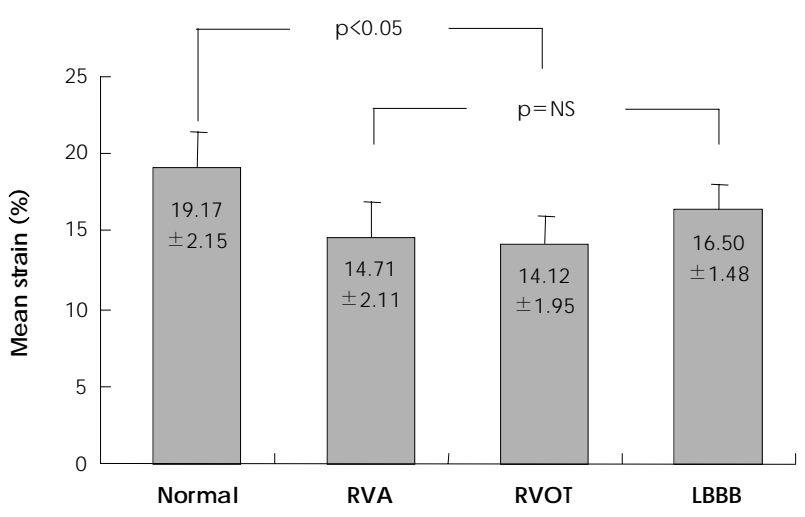

Fig. 3. Mean strain for each group. RVA/RVOT: right ventricular apical and outflow tract pacing, LBBB: left bundle branch block.
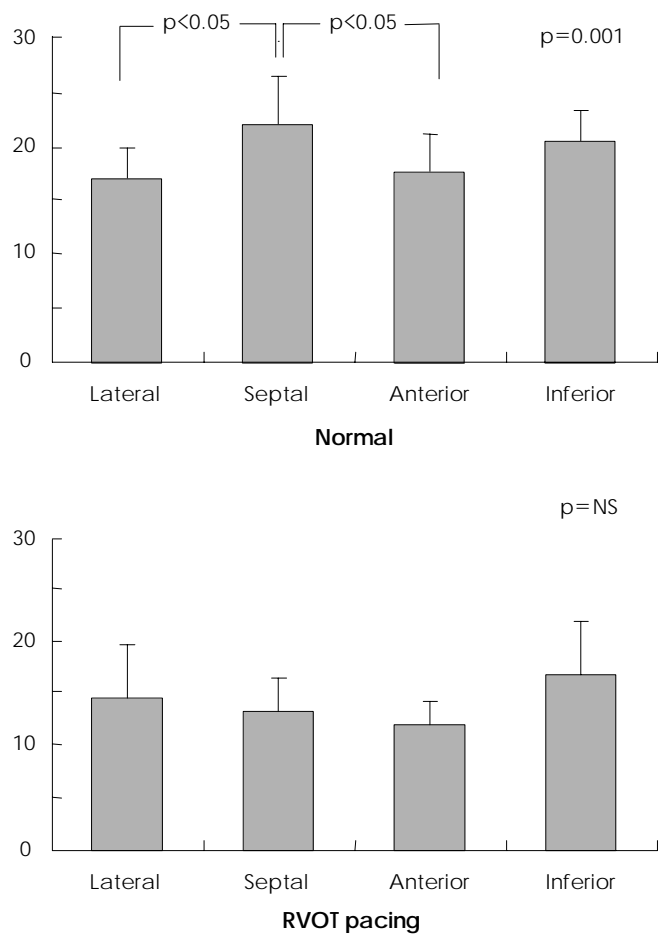

group (Table 2). When the dispersion of the CEP time was corrected for the heart rate, the difference between the RV pacing and LBBB groups nearly disappeared; the dispersion of the CEP time in the aforementioned groups was significantly higher than that in the normal group (Fig. 5). In addition, the standard deviation of the CEP time in the LBBB group was significantly increased compared to those in the normal and RVA pacing groups. However, there was no statistically significant difference in SD between the normal and RV pacing groups (Fig. 6).

\section{Discussion}

The principal finding in this study was that the degree of regional myocardial contraction and dyssynchrony assessed by Doppler tissue strain imaging was similar in the RVA and RVOT pacing and LBBB groups. Based on these findings, RVOT pacing may not be a more favorable alternative and may result in LV dysfunction similar to that observed in patients with RVA pacing or

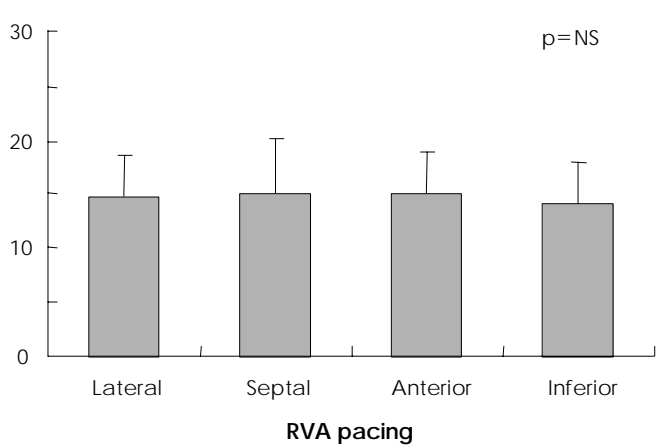

$\mathrm{p}=\mathrm{NS}$

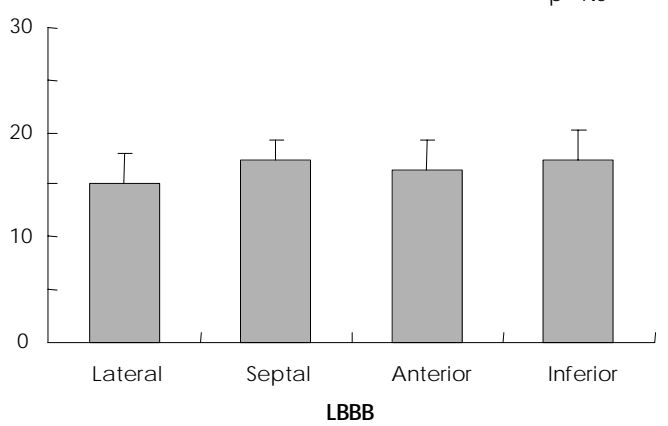

Fig. 4. Mean strain in each wall. RVA/RVOT: right ventricular apical and outflow tract pacing, LBBB: left bundle branch block.

Table 2. Comparison of CEP time and dispersion of CEP times in each groups

\begin{tabular}{|c|c|c|c|c|c|}
\hline & Normal & RVA pacing & RVOT pacing & LBBB & $\mathrm{p}$ \\
\hline CEP time (msec) & $389.1 \pm 28.8$ & $330.4 \pm 28.5^{*}$ & $347.6 \pm 44.1$ & $459.2 \pm 61.0^{* \dagger キ}$ & $<0.001$ \\
\hline CEP time corrected by HR $\left(\mathrm{msec}^{1 / 2}\right)$ & $12.7 \pm 0.9$ & $14.8 \pm 1.2$ & $15.5 \pm 2.0^{*}$ & $15.2 \pm 3.5^{*}$ & 0.004 \\
\hline SD of CEP times (msec) & $45.9 \pm 10.9$ & $45.4 \pm 16.5$ & $52.6 \pm 17.8$ & $70.3 \pm 23.5^{* \dagger}$ & 0.007 \\
\hline Dispersion of CEP times (msec) & $119.9 \pm 18.5$ & $175.1 \pm 51.6$ & $165.3 \pm 57.9$ & $230.0 \pm 68.5^{* \neq}$ & $<0.001$ \\
\hline Dispersion of CEP time corrected by HR $\left(\mathrm{msec}^{1 / 2}\right)$ & $3.92 \pm 0.60$ & $7.83 \pm 2.31^{*}$ & $7.39 \pm 2.59^{*}$ & $7.58 \pm 2.48^{*}$ & $<0.001$ \\
\hline
\end{tabular}

$*<0.05$ compared to the normal group, $\dagger<0.05$ compared to the RVA pacing group, ${ }^{*}<0.05$ compared to the RVOT pacing group. RVA/ RVOT: right ventricle apical/outflow tract, LBBB: left bundle branch block, CEP: compression/expansion crossover point, SD: standard deviation 


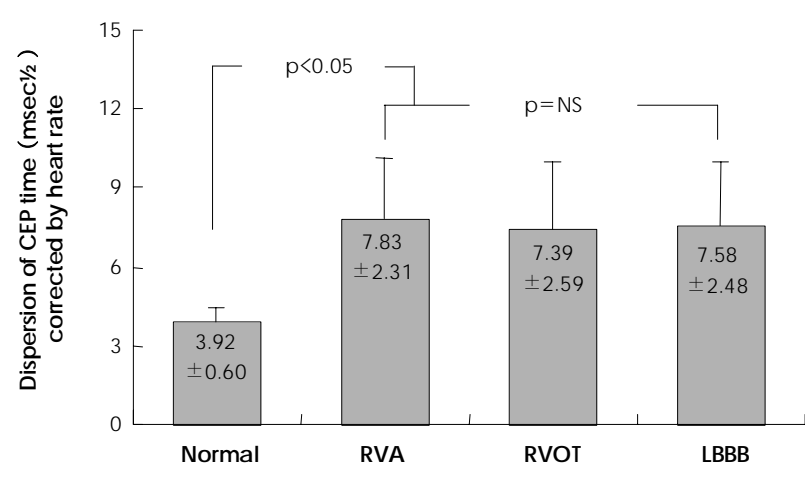

Fig. 5. Mean dispersion of compression/expansion crossover point (CEP) time. RVA/RVOT: right ventricular apical and outflow tract pacing, LBBB: left bundle branch block.

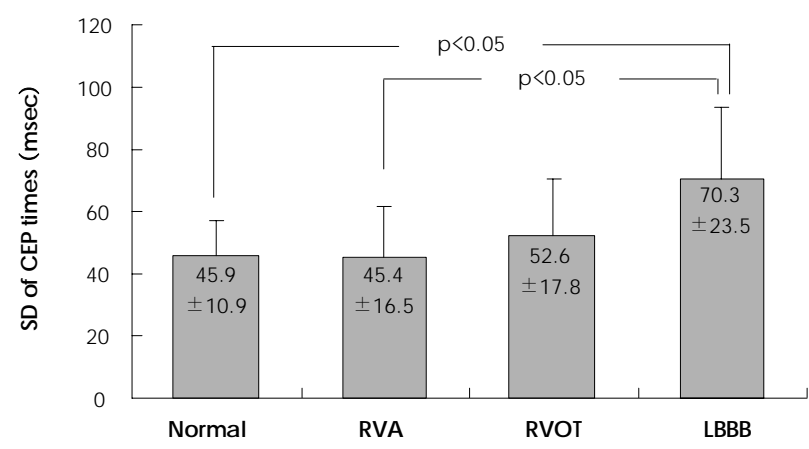

Fig. 6. Standard deviations (SD) of the mean compression/expansion crossover point (CEP) times in each group. RVA/RVOT: right ventricular apical and outflow tract pacing, LBBB: left bundle branch block.

\section{those with LBBB.}

Several authors have reported conflicting findings concerning the acute and chronic differences in hemodynamic effects between RVA and RVOT pacing. ${ }^{15-19)} \mathrm{A}$ pooled analysis of nine prospective studies evaluating the hemodynamic effects of RVOT pacing in $217 \mathrm{pa}-$ tients indicated a modest but significant hemodynamic benefit compared with RVA pacing. ${ }^{15)}$ Giudici et al. ${ }^{16)}$ reported that the cardiac output improved at the time of implantation with RVOT pacing, from $6.6 \mathrm{~L} / \mathrm{min}$ at the apex to $7.8 \mathrm{~L} / \mathrm{min}$ at the outflow tract, a $19 \%$ increase. In contrast, Buckingham et al. ${ }^{18)}$ demonstrated an insignificant increase in the $\mathrm{dP} / \mathrm{dt}$ during single site RVOT pacing, as compared to single site RVA pacing. Victor et al. ${ }^{19)}$ found neither a substantial change in the functional class nor a hemodynamic benefit during RVOT pacing as compared to RVA pacing during midterm follow-up.

There are two possible explanations for these mixed results. First, the position of the lead in the RVOT could have influenced the results. Most of the previous studies described the lead position using X-ray images, and only some of them identified the final lead position using the pacing-induced QRS width. ${ }^{1517)}$ The different investigators paced at different sites in the RVOT. Tse ${ }^{17)}$ and Stambler et al. ${ }^{20)}$ reported that the QRS width in RVOT pacing was shorter compared to that in RVA pacing.
However, there was a different QRS width for RVOT pacing in the two studies (134 ms vs. $167 \mathrm{~ms}$, respectively). Increasing distance of the RV pacing site from the His-Purkinje system causes prolongation of the QRS interval and greater intramyocardial conduction time, which may provoke LV dyssynchrony. ${ }^{21)}$ Riedlbauchova et $\mathrm{al}^{22}{ }^{22}$ suggested that the ideal pacing position seems to be the mid-septum, where the earliest endocardial signal can be observed, often with a potential from the right bundle. This was reflected by a narrow QRS complex as compared to RVOT pacing. In our study, we positioned the pacing lead in the high septum using fluoroscopic and ECG morphologic guidance, involving a LBBB and inferior axis. However, the QRS width for RVOT pacing was $163 \mathrm{~ms}$.

Second, pacing-induced acute changes in hemodynamic performance do not necessarily predict hemodynamic improvement during long-term pacing. Like the adverse hemodynamic and clinical effects of spontaneous LBBB, the iatrogenic variety of the LBBB produced by conventional RVA pacing technique employed in permanent cardiac pacing may be equally harmful. ${ }^{23)}$ Our study showed a similar degree of contraction, assessed by Doppler tissue strain imaging, among the RVOT pacing, RVA pacing, and LBBB groups. This suggests that RVOT pacing-induced LBBB exhibited similar contractile dysfunction compared to that seen in the idiopathic LBBB group.

In the present study, we measured the dispersion of the CEP time in the 12 segments using Doppler tissue strain imaging as the index of dyssynchrony. Patients with heart failure have concurrent electrical delay on the surface ECG, mainly in the form of LBBB. This ventricular conduction disturbance changes the LV contraction pattern, resulting in dyssynchronized intra- and inter-ventricular contraction and further impairing systolic performance. In addition, abnormal myocardial shearing forces and stress vectors may manifest as abnormal contractility and dyssynchrony. ${ }^{24)}$ Doppler tissue imaging, strain rate, and strain have been demonstrated to be useful tools in evaluating myocardial dyssynchrony. ${ }^{25-27)}$ Doppler tissue strain imaging can measure the local myocardial shortening and lengthening and is less sensitive to segment tethering and translation than is tissue Doppler imaging. ${ }^{28)}$ The strain image may be used as a more precise tool for quantifying ventricular contractility and dyssynchrony.

There are a few limitations to this study. We chose a 3 -minute pacing period. It is possible that the results may have been different if a longer period had been allowed for conditions to be established. However, two papers ${ }^{18229)}$ which measured echocardiographic and hemodynamic parameters after pacing by using catheters at different sites, also used 2- or 3-minute pacing protocols. Additionally, a relatively small number of pa- 
tients was analyzed in this study. A large-scale, longterm follow-up study will be required in the future. Another limitation of our study is that dyssynchrony was measured using only Doppler tissue strain imaging instead of strain rate imaging. Acute findings in dyssynchrony and regional contraction may be related to chronic adverse responses. However, it is unclear if these acute findings predict long-term clinical effects.

In conclusion, the present study suggests that RVOT pacing, which is characterized by a wide QRS width, is associated with significant mechanical dyssynchrony and is not a viable alternative to $\mathrm{RV}$ apical pacing. As a result, RVOT pacing may have an adverse effect on long-term LV function similar to that observed with RV apical pacing.

\section{Acknowledgments}

We thank Mr. John Martin for his linguistic review. This work was supported by grant No. RTI04-01-01 from the Regional Technology Innovation Program of the Ministry of Commerce, Industry, and Energy (MOCIE).

\section{REFERENCES}

1) Wilkoff BL. The Dual Chamber, and VVI Implantable Defibrillator (DAVID) Trial: rationale, design, results, clinical implications and lessons for future trials. Card Electrophysiol Rev 2003; 7:468-72.

2) Moss AJ, Zareba W, Hall WJ, et al. Prophylactic implantation of a defibrillator in patients with myocardial infarction and reduced ejection fraction. N Engl J Med 2006;346:877-83.

3) Lee MY, Rho TH, Yoo KD, et al. The relationship between QRS duration and $L V$ systolic function in paced patients. Korean Circ J 2001;31:327-34.

4) Tse HF, Lau CP. Long-term effect of right ventricular pacing on myocardial perfusion and function. J Am Coll Cardiol 1997;29: 744-9.

5) Cha KS, Min JJ, Kim JH, et al. Alterations in myocardial perfusion and regional wall motion in patients with permanent pacemaker. Korean Circ J 1998;28:506-15.

6) McGavigan $\mathrm{AD}$, Mond HG. Selective site ventricular pacing. Curr Opin Cardiol 2006;21:7-14.

7) Sweeney MO, Prinzen FW. A new paradigm for physiologic ventricular pacing. J Am Coll Cardiol 2006;47:282-8.

8) Gold MR, Shorofsky SR, Metcalf MD, Feliciano Z, Fisher ML, Gottlieb SS. The acute hemodynamic effects of right ventricular septal pacing in patients with congestive heart failure secondary to ischemic or idiopathic dilated cardiomyopathy. Am J Cardiol 1997;79:679-81.

9) Kim HY, Kim JH, Rho TH, et al. Hemodynamic responses to different ventricular pacing sites and pacing rates in dog. Korean Circ J 1998;28:1605-15.

10) De Cock CC, Meyer A, Kamp O, Visser CA. Hemodynamic benefits of right ventricular outflow tract pacing: comparison with right ventricular apex pacing. Pacing Clin Electrophysiol 1998;21:536-41.

11) Mabo P, Scherlag BJ, Munsif A, Otomo K, Lazzara R. A technique for stable His-bundle recording and pacing: electrophysiological and hemodynamic correlates. Pacing Clin Electrophysiol 1995;18:1894-901.

12) Gorcsan J 3rd, Kanzaki H, Bazaz R, Dohi K, Schwartzman D. Usefulness of echocardiographic tissue synchronization imaging to predict acute response to cardiac resynchronization therapy.
Am J Cardiol 2004;93:1178-81.

13) Dohi K, Suffoletto MS, Schwartzman D, Ganz L, Pinsky MR, Gorcsan J 3rd. Utility of echocardiographic radial strain imaging to quantify left ventricular dyssynchrony and predict acute response to cardiac resynchronization therapy. Am J Cardiol 2005;96:112-6.

14) Bazett HC. An analysis of the time-relation of electrocardiograms. Heart 1920;7:353-70.

15) De Cock CC, Giudici MC, Twisk JW. Comparison of the haemodynamic effects of right ventricular outflow-tract pacing with right ventricular apex pacing: a quantitative review. Europace 2003; 5:275-8.

16) Giudici MC, Thomburg GA, Buck DL, et al. Comparision of right ventricular outflow tract and apical lead permanent pacing on cardiac output. Am J Cardiol 1997;79:209-12.

17) Tse HF, Yu C, Wong KK, et al. Functional abnormalities in patients with permanent right ventricular pacing: the effect of sites of electrical stimulation. J Am Coll Cardiol 2002;40:1451-8.

18) Buckingham TA, Candinas R, Attenhofer $C$, et al. Systolic and diastolic function with alternate and combined site pacing in the right ventricle. Pacing Clin Electrophysiol 1998;21:1077-84.

19) Victor F, Leclercq C, Mabo P, et al. Optimal right ventricular pacing site in chronically implanted patients: a prospective randomized crossover comparison of apical and outflow tract pacing. J Am Coll Cardiol 1999:33:311-6.

20) Stambler BS, Ellenbogen $K$, Zhang $X$, et al. Right ventricular outflow versus apical pacing in pacemaker patients with congestive heart failure and atrial fibrillation. J Cardiovasc Electrophysiol 2003;14:1180-6.

21) Rosenqvist M, Bergfeldt L, Haga Y, Rydén J, Rydén L, Owall A. The effect of ventricular activation sequence on cardiac performance during pacing. Pacing Clin Electrophysiol 1996;19:127986.

22) Riedlbauchova L, Kautzner J, Hatala R, Buchingham TS. Is right ventricular outflow tract pacing an alternative to left ventricular/ biventricular pacing? Pacing Clin Electrophysiol 2004;27:871-7.

23) Thambo JB, Bordachar P, Garrigue S, et al. Detrimental ventricular remodeling in patients with congenital complete heart block and chronic right ventricular apical pacing. Circulation 2004; 110:3766-72.

24) Bax JJ, Ansalone G, Breithardt OA, et al. Echocardiographic evaluation of cardiac resynchronization therapy: ready for routine clinical use?: a critical appraisal. J Am Coll Cardiol 2004;44:1-9.

25) Bax JJ, Marwick TH, Molhoek SG, et al. Left ventricular dyssynchrony predicts benefit of cardiac resynchronization therapy in patients with end stage heart failure before pacemaker implantation. Am J Cardiol 2003;92:1238-40.

26) Yu CM, Fung JW, Zhang Q, et al. Tissue Doppler imaging is superior to strain rate imaging and postsystolic shortening on the prediction of reverse remodeling in both ischemic and nonischemic heart failure after cardiac resynchronization therapy. Circulation 2004;110:66-73.

27) Dohi K, Suffoleto MS, Schwarzman D, Ganz L, Pinsky MR, Gorcsan J 3rd. Utility of echocardiographic radial strain imaging to quantify left ventricular dyssynchrony and predict acute response to cardiac resynchronization therapy. Am J Cardiol 2005;96:112-6.

28) Abraham TP, Nishimura RA, Holmes DR Jr, Belohlavek M, Seward JB. Strain rate imaging for assessment of regional myocardial function: results from a clinical model of septal ablation. Circulation 2002;105:1403-6.

29) Blanc JJ, Etienne Y, Gilard M, et al. Evaluation of different ventricular pacing sites in patients with severe heart failure: results of an acute hemodynamic study. Circulation 1997;96: 3273-7. 